\title{
Mars Shot for Nuclear Medicine, Molecular Imaging, and Molecularly Targeted Radiopharmaceutical Therapy
}

\author{
Richard L. Wahl ${ }^{1}$, Panithaya Chareonthaitawee ${ }^{2}$, Bonnie Clarke ${ }^{3}$, Alexander Drzezga ${ }^{4}$, Liza Lindenberg ${ }^{5}$, \\ Arman Rahmim ${ }^{6}$, James Thackeray ${ }^{7}$, Gary A. Ulaner ${ }^{8}$, Wolfgang Weber ${ }^{9}$, Katherine Zukotynski ${ }^{10}$, and John Sunderland ${ }^{11}$ \\ ${ }^{I}$ Mallinckrodt Institute of Radiology, Washington University St. Louis, Missouri; ${ }^{2}$ Department of Cardiovascular Medicine, Mayo \\ Clinic, Rochester, Minnesota; ${ }^{3}$ Research and Discovery, Society of Nuclear Medicine and Molecular Imaging, Reston, Virginia; \\ ${ }^{4}$ Department of Nuclear Medicine, University of Cologne, Cologne, Germany, German Center for Neurodegenerative Diseases, Bonn- \\ Cologne, Germany, and Institute of Neuroscience and Medicine, Molecular Organization of the Brain, Forschungszentrum Jülich, \\ Jülich, Germany; ${ }^{5}$ Molecular Imaging Program, Center for Cancer Research, National Cancer Institute, National Institutes of Health, \\ Bethesda, Maryland; ${ }^{6}$ Departments of Radiology and Physics, University of British Columbia, Vancouver, British Columbia, Canada; \\ Department of Integrative Oncology, BC Cancer Research Institute, Vancouver, British Columbia, Canada; ${ }^{7}$ Department of Nuclear \\ Medicine, Hannover Medical School, Hannover, Germany; ${ }^{8}$ Department of Radiology, Memorial Sloan Kettering Cancer Center, New \\ York, New York, and Molecular Imaging and Therapy, Hoag Cancer Center, Newport Beach, California; ${ }^{9}$ Department of Nuclear \\ Medicine, Technical University Munich, Munich, Germany; ${ }^{10}$ Departments of Medicine and Radiology, McMaster University, \\ Hamilton, Ontario, Canada; and ${ }^{11}$ Departments of Radiology and Physics, University of Iowa, Iowa City, Iowa
}

\begin{abstract}
The Society of Nuclear Medicine and Molecular Imaging created the Value Initiative in 2017 as a major component of its strategic plan to further demonstrate the value of molecular imaging and molecularly targeted radiopharmaceutical therapy to patients, physicians, payers, and funding agencies. The research and discovery domain, 1 of 5 under the Value Initiative, has a goal of advancing the research and development of diagnostic and therapeutic nuclear medicine. Research and discovery efforts and achievements are essential to ensure a bright future for NM and to translate science to practice. Given the remarkable progress in the field, leaders from the research and discovery domain and society councils identified 5 broad areas of opportunity with potential for substantive growth and clinical impact. This article discusses these 5 growth areas, identifying specific areas of particularly high importance for future study and development. As there was an understanding that goals should be both visionary yet achievable, this effort was called the Mars shot for nuclear medicine.
\end{abstract}

Key Words: radiopharmaceutical therapy; artificial intelligence; theranostics; molecular imaging; neuroimaging

J Nucl Med 2021; 62:6-14

DOI: 10.2967/jnumed.120.253450

$\mathbf{N}$ radiopharmaceutical therapy (MTRT) are a result of the integration of science and technologies from a variety of areas of biomedical, physical, and computational sciences. An understanding of biologic processes and diseases represents the foundation and rationale for radiopharmaceutical science. Chemistry and biology underpin the development of radiopharmaceuticals, both

Received Jul. 13, 2020; revision accepted Sep. 23, 2020.

COPYRIGHT (C) 2021 by the Society of Nuclear Medicine and Molecular Imaging. diagnostic and therapeutic. Our detection of signals from radioisotopes depends on physical principles and instrumentation. Complex datasets are increasingly analyzed using advanced computational algorithms, including more advanced machine-learning algorithms. These tools of NM are then applied to clinical challenges and research questions in NM in areas of opportunity in the brain, heart, oncology, and, more broadly, human health and disease. The rapid progress in science in a variety of these scientific-foundational areas offers opportunities for major investments to advance clinical translation of emerging technologies in NM.

Recently, a multidisciplinary group of scientists and physicians from the research and discovery domain of the SNMMI's Value Initiative collaborated to identify and curate the leading initiatives and opportunities in NM and molecular imaging (MI). The society's councils and centers of excellence-namely, the PET Center of Excellence, the Therapy Center of Excellence, and the Center for Molecular Imaging Innovation and Translation-were asked to submit the top opportunities in their area for MI and therapy research. The responses were tabulated and categorized, and experts were selected from the membership of the councils and centers of excellence in each of 5 main areas of opportunity and were invited to expand on the topics of physics, instrumentation, and data sciences; neurologic imaging; cardiac imaging; oncologic imaging; and MTRT. The goal was to identify major opportunities and to help guide society, funding, and industry investments in the field. The data were collected shortly before the coronavirus 2019 pandemic set upon us.

\section{PHYSICS, INSTRUMENTATION, AND DATA SCIENCES FRONTIER}

Continuing advances in imaging equipment and in reconstruction and analysis tools allow better visualization, quantification, and interpretation of imaging studies done today, opening the door for new and deeper discoveries tomorrow. 


\section{Data Science and Artificial Intelligence}

Effective collection and sharing of image and patient data to drive machine learning approaches will strengthen clinical capabilities. There is significant interest in the use of advanced data sciences, including machine learning (especially deep learning) methods, in health care. Although their use provokes uncertain (and sometimes inflated) expectations, significant benefits are expected relative to benchmarked standards of care (1). Systematic efforts may subsequently enable better standardization of data acquisition, reduced radiation dose or scan times, and significantly improved diagnostic, predictive, prognostic and treatment response assessment capabilities. To these ends, the community needs to expand intersocietal collaborations and academic-industry partnerships; promote data sharing; encourage standardization and harmonization initiatives; link imaging studies with clinical reports, laboratory test results, genomic or proteomic assays, and clinical outcomes; and focus on generalizability and critical appraisal of the methods toward translation to routine clinical practice (in connection with regulatory bodies). The focus should not be purely on deep learning methods. For example, in oncology, there is significant potential for the use of radiomics and explicitly defined texture and shape measures, some of which are reproducible and effective (2). There are other clinical application opportunities. Data-driven (markerless, softwarebased) motion detection and associated correction techniques have potential to significantly improve the quality and quantitative accuracy of NM images (an increasingly important topic given improved spatial resolution in scanners). We envision a future where motion correction (including respiratory, cardiac, and gross patient motion) is applied routinely in the clinic. In neurology, observer-independent procedures such as stereotactic normalization and automated voxel-based comparison of patient brain imaging data to healthy control populations are well established. There are now opportunities to develop automated image interpretation algorithms (3). Regarding the high discriminatory accuracy of many methods of nuclear neuroimaging, these tests may form an ideal basis for training of deep learning procedures and translation into clinical practice.

\section{NOTEWORTHY}

- Artificial intelligence and machine learning may enable data acquisition, reduce radiation dose or scan times, and improve the speed and accuracy of image interpretations, leading to improved diagnostic, predictive, prognostic, and treatment response assessments.

- Nuclear imaging is expected to play a greater role in understanding the biology of, diagnosing, and guiding treatments for dementia, movement disorders, epilepsy, multiple sclerosis, brain tumors, and psychiatric diseases.

- Identification of atherosclerotic plaques, myocardial infarction, and progressive heart failure with MI may lead to novel therapeutic approaches to mitigate the inflammatory process.

- New biochemically disease-specific PET radiopharmaceuticals will change the landscape of PET oncology imaging.

- Recent advances in MTRTs will significantly broaden the application of radiopharmaceutical therapy in research and in clinical practice.

- Powered by advances in both quantitative PET and SPECT/ CT, personalized image-based dosimetry will drive MTRT to greater effectiveness while preserving safety.

\section{Novel Imaging Systems}

Clinical applications of PET/MRI systems will become more mainstream; total-body PET/CT systems will define new limits of clinical imaging and pharmacokinetic study; and there will be more development and optimization of dedicated instruments for wider use. The biologic and biochemical development of specifically targeted radiotracers will be augmented by technologic developments in scanners.

Integrated PET/MRI, a developing field in the last decade, is still in its early stages of application and holds potential for improved clinical diagnostic performance in several applications $(4,5)$. Dedicated, diverse resources and thoughtful, synergistic efforts can lead to significant advancements and opportunities for larger-scale deployment.

Total-body PET is now 510(k)-approved and available with a field of view large enough to image the entire body simultaneously (6). The technical advantages of total-body PET include the potential to increase sensitivity by a factor of about 40 . Improved image quality, enhanced detection of smaller lesions, and imaging of radiotracers beyond their currently used time frames, such as ${ }^{18} \mathrm{~F}$ imaging $18 \mathrm{~h}$ after tracer administration, are creating a myriad of new clinical and research opportunities (7). This technology also has the potential for significant reductions in patient doses or substantially decreased imaging times, which should be investigated. Total-body acquisitions over multiple time points for full pharmacokinetic characterization of new tracers are another opportunity.

There is significant scope for development and optimization of other instruments with high sensitivity, spatial resolution, or temporal resolution for specific applications and study of prevalent diseases. Opportunities exist for optimized pediatric imaging and for design of wearable, organ-specific or patient-configurable PET or SPECT imaging systems. For example, smaller brain-specific imaging devices may become critical in view of the increasing emphasis on early detection of diseases such as dementia (including Alzheimer disease) (8). Overall, dedicated imaging systems could provide significant cost or performance benefits and enable applicability to wider populations.

\section{Pharmacokinetic Quantitation}

Advances in scanner, reconstruction, and artificial intelligence technology push physiologically and biologically based imaging biomarkers from the research lab to the clinic.

Current clinical protocols are optimized primarily for qualitative assessment (as opposed to fully quantitative assessment). Yet, radiopharmaceutical distribution is a dynamic process with substantial pharmacokinetic variations between tumors and normal organs and among patients. Assessment of such dynamic processes has the potential to improve clinical diagnostic performance (9). Dynamic imaging enables quantitative analysis (e.g., via kinetic modeling) and generation of quantitative parametric data or images. Thus far, quantitative evaluation of myocardial blood flow in coronary artery disease evaluation has made its way into the clinic. However, there is a broad opportunity for expanded application in oncology and neurology. Here, dynamic imaging can generate parametric images in addition to conventional SUVs from a single imaging session optimized to the clinical workflow (10). This task can be accomplished in single-bed or multibed imaging and in fixed versus organ-specific systems. Given ongoing technical developments, advanced image reconstruction methods, and application of artificial intelligence to lowcount datasets, dynamic images of increasing quality and quantitative 
accuracy are being obtained. Aided by this progress, there is a real opportunity for exploring shorter scan times in which physiologically or biologically meaningful kinetic parameters are generated as effective imaging biomarkers of disease, opening up venues to add significant value in a clinical setting.

\section{NEUROLOGIC IMAGING}

Diseases of the brain and nervous system are increasingly prevalent, especially in an aging population. There have been substantial investments in basic and clinical science in the areas of dementia, movement disorders, epilepsy, multiple sclerosis, brain tumors, and psychiatric diseases, among others. These investments are leading to a newer understanding of different brain diseases and new therapies. Nuclear imaging is expected to play a greater role in understanding the biology of, diagnosing, and monitoring treatments for these diseases.

\section{Imaging of Neuronal Function}

Imaging neuronal function and dysfunction has been a domain of ${ }^{18}$ F-FDG PET for more than 4 decades; however, movement to new synaptic density measurements may be appropriate for certain indications. ${ }^{18}$ F-FDG PET can still be considered the Swiss army knife of brain imaging in NM, not only because the tracer is widely accessible and relatively affordable (and reimbursed for some indications in several countries, including the United States) but also because ${ }^{18}$ F-FDG PET imaging of the brain provides highly reliable and sensitive information on the extent and location of neuronal dysfunction in a large spectrum of disorders of the central nervous system, ranging from epilepsy to neurodegeneration (11-13). However, some limitations apply to the tracer. One is the lack of information on the underlying cause of changes in neuronal function (14). Other limitations stem from the observation that neuronal activity may not necessarily reflect neuronal health $(15,16)$. Some of these limitations may not apply to imaging of synaptic density. Recently, several promising tracers have been introduced that target mainly the synaptic vesicle proteins, which may allow measurement of synaptic density in vivo in the human brain (17-19). These novel tools may bear great potential to improve understanding of differences between neuronal activity and synaptic density in the healthy and the diseased brain (e.g., neurodegeneration or epilepsy). Furthermore, they may allow for closely following the effects of specific therapies.

\section{Imaging of Protein Pathology}

The development of tools for MI of amyloid deposits has been one of the most visible and game-changing achievements in neuroimaging, NM, and even in neuroscience altogether in recent years (20). The opportunity to detect a pathogenomic molecular pathology in the brain in vivo without the need for a biopsy or invasive procedure has significantly advanced research in the field of neurodegeneration, particularly regarding Alzheimer disease. Numerous publications have considerably impacted the understanding of these disorders, regarding the onset and course of disease or the interaction between pathology and dysfunction (21). Inclusion of patients in modern Alzheimer disease-related treatment trials is already based predominantly on the results from amyloid imaging, and this method may play a crucial role as a gatekeeper as soon as (hopefully) the first disease-modifying drugs arise. However, amyloid deposits represent only one type of protein aggregation, and it is commonly assumed the neurodegenerative disorders in general are based on the deposition of different types of pathologic proteins in the brain. Thus, great efforts are currently invested in the development and evaluation of tracers for tau, synuclein, and several others that may come into focus in the future (22). This endeavor is clearly challenging and, to be successful, will require considerable future commitment and investment. Importantly, a first compound for imaging tau aggregates ( ${ }^{18} \mathrm{~F}$-flortaucipir [Tauvid; Lilly]) has recently been approved by the Food and Drug Administration (FDA) (23).

\section{Imaging of Inflammation}

Neuroinflammatory processes are increasingly implicated in a multitude of disorders of the central nervous system and may play a crucial role in many of them. These include not only primarily immunogenic disorders such as multiple sclerosis but also brain tumors, neurodegenerative disorders, psychiatric disorders (e.g., schizophrenia or depression), brain trauma, and ischemia. Imaging neuroinflammation not only is of great interest to potentially clarify its influence on the development and course of disease but could represent a most attractive target for therapeutic interventions. Tracer development for imaging inflammation by targeting the translocator protein has been hampered for many years by low specific signal, the high interindividual variability in physiologic binding affinity (high, intermediate, and low binders), or limited availability due to ${ }^{11} \mathrm{C}$-labeling (24). However, although novel and improved approaches to imaging translocator protein tracers are being developed, tracers targeting other mechanisms involved in neuroinflammation (e.g., expression of the cyclooxygenases in the brain) may open interesting opportunities to investigate the role of neuroinflammation and potentially introduce imaging of neuroinflammation into clinical application $(25,26)$.

\section{Imaging of Neurotransmission}

One of the extraordinary strengths of MI is the ability to visualize the function and integrity of transmitter systems in the brain through tracers specifically reflecting the density of neuroreceptors or transporters. Only NM methods provide this type of noninvasive insight into cerebral neurochemistry on the molecular level. This potential is still not substantially exploited accordingly with regard to clinical purposes. So far, imaging of the presynaptic dopaminergic system in the diagnosis of Parkinsonian syndromes is wellestablished $(27,28)$. However, numerous disorders of the brain are fundamentally based on a disturbed balance of the neuroreceptor/ transmitter system, including neuropsychiatric disorders such as depression and schizophrenia, as well as neurodegeneration, addiction, and personality disorders. It appears obvious that greater effort is needed to further establish applicable and accessible approaches to neuroreceptor imaging in clinical routine. The value of these methods needs to be tested so that we can deepen our understanding of the involved pathomechanisms and potentially improve early diagnosis, prediction, therapy selection, and response monitoring. A prominent example may be the complex glutamatergic system (29). Glutamate is the predominant excitatory transmitter in the brain. Glutamate receptors are hypothesized to play a key role in both fast and slow excitatory neurotransmission (e.g., learning and neuroplasticity) in the healthy brain and also to be involved in numerous pathologic processes such as epilepsy, addiction, brain trauma, and neurodegeneration. Glutamate receptor imaging may be of high value to potentially guide personalized therapy and is currently an open and exciting area for future exploration.

\section{CARDIAC IMAGING}

Heart disease remains an immense public health issue. Heart disease is the leading cause of death for men, women, and people 
of most racial and ethnic groups in the United States, accounting for an estimated 1 in 4 deaths. Imaging and understanding the heart and blood vessels are an area of great opportunity for NM.

\section{Inflammation}

In migrating from ${ }^{18} \mathrm{~F}-\mathrm{FDG}$-based inflammation imaging to more target-specific inflammation processes in cardiovascular disease, specific opportunities for MI include identification of atherosclerotic plaque, myocardial infarction, and progressive heart failure. Novel therapeutic approaches aim to mitigate the inflammatory process and facilitate endogenous healing. Previously, capitalizing on the glucose avidity of activated inflammatory leukocytes, ${ }^{18}$ F-FDG has been used to image and characterize inflammation in vasculature and myocardium, providing prognostic information on functional outcome (30). However, myocardial ${ }^{18} \mathrm{~F}$-FDG imaging suffers from several limitations as a nonspecific marker. Radiopharmaceuticals specifically targeted to inflammatory cells and substrates are a priority. Several alternative targets highly expressed by activated inflammatory cells have been proposed, including chemokine receptors, somatostatin receptors, mitochondrial translocator protein (31), and receptors on fibroblasts, among others. The intersection between inflammation and fibrosis has also gained interest as a diagnostic and therapeutic target $(32,33)$. Novel, specifically targeted radiotracers have been evaluated in animal models of myocardial infarction and atherosclerosis, with some small-scale clinical evaluation performed (32). Using a common target for imaging and therapy is particularly attractive, might facilitate identification of appropriate patient populations and guidance of antiinflammatory therapeutic decisions, and could be exploited as a surrogate endpoint for clinical drug trials (34).

\section{Flow Quantification}

Improved software and tracers with flow-imaging properties that are closer to ideal will drive more robust flow quantification and greater clinical utility. Myocardial perfusion imaging will remain a dominant component of nuclear cardiology. Greater accuracy of clinical assessment and absolute quantification continue to be top priorities. Deep machine learning may optimize and streamline nuclear cardiology assessment using SPECT (35), but further refinement is needed for routine application. Accurate and reproducible flow quantification using SPECT is desirable, with some centers reporting the feasibility of flow measurements with timing efficiency of cadmium-zinc-telluride detectors and favorable comparison to PET (36). Further clarification will be necessary, but this is an area ripe for development.

PET flow quantification, although more mature than SPECT, also requires further refinements in its application in the clinical setting. PET flow quantification is becoming increasingly common in the clinic, and its diagnostic and incremental prognostic values are well established. However, nonlinearities of radiotracer uptake at high flow are a challenge, as are inconsistent results between software packages, differences in flow reserve measurements, and differences between radiotracers (37). Further careful science would broaden clinical application. In addition, broader clinical application of PET myocardial perfusion imaging may require development of new cost-effective PET perfusion tracers with superior biologic and imaging characteristics, including a high extraction fraction, a short positron range, longer physical halflives (for commercial distribution), and a high correlation between myocardial uptake and coronary blood flow without redistribution (37). Clinical development of such agents should remain a top priority for the field and will promote dissemination and standardization of the methodology.

\section{Cardiac Amyloidosis}

Old SPECT and new PET radiopharmaceuticals may drive this new clinical application, but fundamental mechanistic questions exist, and therapeutic response assessment applications require validation.

After the successful deployment of ${ }^{11} \mathrm{C}$-Pittsburgh compound B and analogs for imaging of $\beta$-amyloid, there has been rising interest in applying similar tracers to amyloidosis in the heart. The spatial resolution of PET is particularly beneficial for amyloidosis, as the distribution pattern in the left ventricle is relevant to accurate diagnosis, particularly for amyloid subtypes (38). New-generation amyloid tracers have been evaluated, and further testing may increase the translation of these techniques.

The clinical utility of bone-seeking radiotracers in diagnosing transthyretin-related cardiac amyloidosis has recently been established (39). Questions remain on the utility of this technique in identifying cardiac involvement in other transthyretin-related mutations, on the generalizability to diverse populations, and on the ability of the technique to monitor disease progression over time. Finally, the mechanism by which these bone-seeking tracers binds to amyloid transthyretin amyloidosis more than light chain amyloid fibrillar deposits remains to be elucidated. With effective treatments now available for transthyretin-mediated amyloidosis, the optimal use of imaging to diagnose and guide therapies represents a major opportunity.

\section{Imaging of Infection}

Highly specific radiopharmaceuticals targeting infection imaging could replace current technologies in the myocardium and throughout the body and complement anatomic imaging methods.

Although ${ }^{18} \mathrm{~F}$-FDG has been functional in defining localized enrichment of activated leukocytes, it cannot distinguish between sterile inflammation and infection. With the rising prevalence of implantable devices and in-hospital infections, reliable identification of bacterial infection by noninvasive imaging is desirable. The novel radiotracers specifically target proteins expressed solely by bacterial cells, such as the maltodextrin transporter, folates, siderophores, or proteins involved in cell wall synthesis. For example, labeled analogs of maltose sugars, including ${ }^{18} \mathrm{~F}$-maltotriose, have been characterized in preclinical infection models and myocarditis (40), with clinical translation imminent. In addition to specificity, accurate and reproducible quantitation will be paramount for the successful rollout of candidate tracers, as the target patient population will inevitably be treated with antibiotics. Although cardiology-based infections have been a focus in the imaging of bacterial infections, applications in other fields for infection should develop in parallel. Opportunities for imaging viral diseases, such as coronavirus disease 2019-related myocarditis, also are emerging.

\section{Clinical Outcome Studies}

The growth of molecular cardiac imaging will depend on rigorous prospective clinical trial designs demonstrating clinical impact. As in other areas of NM, having well-designed, prospective clinical trials with relevant hard outcomes is essential to boost the quality of our evidence base to inform decision making. The large number of patients with cardiac disease in the United States lends itself to careful, well-designed clinical studies. Such studies are essential for public health decisions and for the appropriate integration of innovative 
imaging into clinical care pathways. Such studies should include quality-of-life assessments and hard clinical endpoints and, ideally, should include collecting cost data.

\section{ONCOLOGIC IMAGING}

Imaging of cancer has been a growth area in MI for 2 decades. However, the field is experiencing a paradigm shift in which more general imaging agents such as ${ }^{18} \mathrm{~F}$-FDG are being replaced by more disease-specific or target-specific radiopharmaceuticals. The FDA's approval of new, more biochemically disease-specific (e.g., neuroendocrine cancer, prostate cancer, and breast cancer) PET radiopharmaceuticals is beginning to change the landscape of PET oncology imaging. Besides generating higher sensitivities and specificities, these tracers precisely identify the presence and magnitude of therapeutic targets for precision therapeutic approaches. Capitalizing on this capability has immense potential for propelling MI into the forefront of the development of novel therapies due to both medical and economic efficiencies.

\section{Use of the Full Potential of ${ }^{18}$ F-FDG PET}

More robust application of quantitative ${ }^{18} \mathrm{~F}-\mathrm{FDG}$ PET imaging, particularly in response to therapy, will help maximize its clinical potential. Currently, clinical oncologic PET focuses primarily on the use of ${ }^{18} \mathrm{~F}$-FDG for the diagnosis, staging, and treatment response assessment of cancer. ${ }^{18}$ F-FDG PET, assessing cancer metabolism, can measure cancer activity and burden at multiple time points (41) and is commonly used to determine whether a therapy has been effective. A considerable opportunity for ${ }^{18}$ F-FDG PET includes early assessment of the efficacy of cancer therapies to help aid in intensification or deintensification of therapy protocols soon after cancer therapy has started, as opposed to after it has been completed. The success of expanding ${ }^{18}$ F-FDG's utility in this area will be contingent on well-controlled prospective therapeutic clinical trials using ${ }^{18} \mathrm{~F}-\mathrm{FDG}$, with endpoints guided by quantitative metrics. Development and validation of automated software tools and standardized approaches to data collection and analysis, including newer metrics such as metabolic tumor volume and metabolic tumor burden, are likely necessary prerequisites to successful implementation.

\section{New PET Imaging Agents for Cancer}

Prostate. New radiopharmaceuticals specifically targeting prostate cancer open the door for MI to play a central role in disease management in the future. Although ${ }^{18} \mathrm{~F}-\mathrm{FDG}$ imaging remains the workhorse of clinical PET oncology imaging, ${ }^{18}$ F-FDG imaging has been of limited utility in prostate cancer. However, MIbased prostate cancer detection made immense strides forward with the FDA approvals of ${ }^{11} \mathrm{C}$-choline (42) and ${ }^{18} \mathrm{~F}$-fluciclovine (Axumin; Blue Earth Diagnostics) (43). Prostate cancer management is poised to take another leap forward with the development and downstream approval of prostate-specific membrane antigen (PSMA) targeting agents. PSMA-targeted PET tracers are quickly being recognized as the most sensitive method of detecting prostate cancer and its metastases (44-47). The targeting mechanism of these PSMA-based radiopharmaceuticals lends itself not only to diagnostic imaging but also to identification of patient-specific molecular targets for MTRT and more traditional chemotherapeutics $(48,49)$. In this model, noninvasive whole-body PET is used not just to monitor therapy response but to select the therapies a cancer patient receives, possibly including observation or active surveillance
Breast. New radiopharmaceuticals targeting receptor expression in breast cancer change the paradigm for patient management. Medical oncology is evolving into a field of therapeutic agents based on specific molecular targets, allowing targeted therapy of tumor cells expressing the appropriate target. Breast cancer subtyping, based on receptor status, is an outstanding candidate for MI characterization to guide therapy. The recent FDA approval of ${ }^{18} \mathrm{~F}$-fluoroestradiol has opened the door to accurate identification of estrogen-positive disease on a wholepatient level, which will soon have a substantial impact on patient management (50). Potential clinical applications of ${ }^{18} \mathrm{~F}$-fluoroestradiol include selection of patients for estrogen receptor-targeted therapy $(51,52)$, staging of breast tumors with lower glucose metabolism such as lobular breast cancers $(53,54)$, estrogen receptor evaluation of metastases that are difficult to biopsy (55), and problem solving for ambiguous findings in patients with estrogen receptor-positive tumors (56). New PET tracers targeting progesterone receptor expression are also being developed $(57,58)$. Further, trastuzumab and pertuzumab are antibodies that target human epidermal growth factor receptor 2 (HER2), found on a subset of breast malignancies. Treatment with these and other HER2-targeted therapies has prolonged survival in patients with HER2-positive breast cancer. However, evidence is now accumulating that HER2 receptor expression may change over time and space (59-61), and inaccurate knowledge of HER2 receptor status in tumors may lead to suboptimal use of HER2-targeted therapies. Noninvasive imaging with HER2-targeted radiotracers (62-65) would allow for whole-body evaluation of HER2 expression and help select appropriate breast cancer patients for HER2-targeted therapy even among patients with previously presumed HER2-negative disease (66).

Other Molecular Targets in Oncology. The future holds promise for a growing list of specifically targeted PET tracers. Alterations in the stroma of tumor masses has led to novel opportunities for imaging and therapy of a wide array of malignancies. Multiple PET tracers targeting fibroblast activation protein have been developed and demonstrate excellent tumor-to-background contrast ratios in many tumor types (67-72), with downstream opportunities as therapeutic targets. Additionally, there are a growing number of immuno-PET agents in clinical trials. For example, daratumumab is an antibody that targets CD38, a receptor found on most myeloma cells. Daratumumab labeled with positron-emitting isotopes has demonstrated the ability to image myeloma cells in mouse models and has completed first-in-humans trials (73-76). Novel ${ }^{124}$ I-metaiodobenzylguanidine PET tracers that specifically target adrenergic cells for improved imaging of neuroblastoma are in early clinical trials and may help guide selection of patients for ${ }^{124}$ I-metaiodobenzylguanidine-targeted therapies (77). Thus, whereas nonspecific metabolic imaging using ${ }^{18} \mathrm{~F}-\mathrm{FDG}$ is by far the most commonly applied application of PET today, a new era of targeted MI will greatly expand PET into a noninvasive, wholebody assay for selection of optimal targeted therapies for individual patients in a wide array of malignancies.

\section{Immune Modulation Therapy}

Monoclonal antibodies and other small molecules disrupting immune checkpoints on $\mathrm{T}$ cells have drastically changed the landscape of therapy for many types of cancer. In addition, genetically engineered $\mathrm{T}$ cells and other types of cells are being applied to treat a variety of neoplasms. These therapies can be remarkably effective but are expensive and not devoid of toxicities. Determining whether these therapies are likely to work-and if they have worked - is an incompletely answered question for ${ }^{18} \mathrm{~F}$-FDG PET, as it is challenged by imaging metabolic changes in both tumor and host response (78). There 
are great opportunities to use MI to better interrogate tumors for several variables: their metabolic changes in response to immunotherapy, their levels of checkpoint inhibitor targets in tumors, and their lymphocyte content (79-81). A first-in-humans trial of the anti-CD8 minibody ${ }^{89} \mathrm{Zr}-$ IAB22M2C has demonstrated successful targeting of CD8-positive Tcell-rich tissues, including infiltrates in tumors, and further trials are under way to determine whether imaging can inform immunotherapy decisions (82). There are other targets of possible interest as well. In addition, imaging of the presence and activity of genetically reengineered cells in vivo to predict efficacy represents a major opportunity for MI. Better imaging in immunotherapy represents a great opportunity to improve patient management and preserve economic resources.

\section{MTRT AND THERANOSTICS}

MTRT is a rapidly growing paradigm-shifting approach that has the potential to change the nature and scope of NM practice. By using the same molecular target, it further tightens the connection between imaging and therapy of cancer. The greatest recent success of this strategy was in neuroendocrine malignancies. The recent FDA approval of ${ }^{68} \mathrm{Ga}$-DOTATATE, ${ }^{68} \mathrm{Ga}$-DOTATOC, and ${ }^{64} \mathrm{Cu}$-DOTATATE-tracers targeting somatostatin receptors on neuroendocrine cells-has allowed not just improved detection of neuroendocrine tumors but selection of patients for targeted somatostatin receptor therapy with ${ }^{177}$ Lu-DOTATATE (which has shown excellent results, including markedly prolonged progression-free survival) $(83,84)$. For prostate cancer, PET with tracers targeting PSMA is developing into a tremendous diagnostic tool for localizing biochemical disease recurrence (85). There is great promise for these agents to be approved for selection of appropriate local and hormonal therapies (86), as well as for selection of patients for both radioactive and nonradioactive PSMAtargeted therapies $(87,88)$. Critically, PSMA and somatostatin receptors are not the only molecular targets that can be exploited. Other potential therapeutic targets have been identified and are in early-phase development by both academia and industry (89).

MTRT is a form of precision medicine. A nonspecific toxic agent (e.g., a radioisotope emitting $\beta$-radiation, such as ${ }^{177} \mathrm{Lu}$ ) specifically accumulates in the tumor tissue by virtue of the biochemical targeting properties of the molecule to which the radioisotope is attached. Patients are not selected by ex vivo analyses of the tumor tissue or blood biomarkers but instead by nuclear imaging that demonstrates and quantifies tumor radioactivity uptake. Although the target for radionuclide therapy can be a protein that is critical for the cancer cell, this is not necessary. Instead, a high concentration of the biochemical target in the tumor in combination with a relatively low expression by normal organs are key determinants of a promising target for radionuclide therapy.

The recent advances in MTRT provide the foundation for exciting clinical, translational, and basic research that has the potential to significantly broaden the application of radionuclide therapy in clinical practice and improve patient outcomes.

\section{Optimization of the Dose and Dose Schedule for MTRT}

Prospective studies to understand the impact of activity and dosing intervals on tumor response and toxicity will drive informed, optimized dosing schedules. The dose of radiopharmaceuticals, both radioactive and mass dose, as well as the dose schedule, is currently largely empiric. Formal dose escalation studies, which are standard for oncologic drugs, are generally lacking. Since hematologic and other side effects of peptide-based
MTRT have generally been mild, higher amounts of activity, shorter dosing intervals, or larger numbers of treatment cycles appear feasible and should be studied systematically. These studies must also investigate the long-term toxicity of MTRT. More safety data are critically necessary to advance application of MTRT to earlier tumor stages, as this toxicity may limit the efficacy of the treatment. Dose escalation using stem cell transplantation approaches is feasible and warrants further study as these methods improve.

\section{Personalized Dosimetry}

Powered by advances in both quantitative PET and SPECT/CT, personalized image-based dosimetry drives MTRT to greater effectiveness. With exciting new developments and the expanded use of MTRT, application of patient-specific (personalized) dosimetry may be critical to optimize efficacy while controlling normal-tissue toxicities (90). Substantial efforts are needed to systematically study the outcome of therapies based on actual delivered absorbed doses to tumors and normal tissue through prospective, controlled clinical trials. Efforts are also needed to usher in the opportunity to adjust the dose for optimized delivery of therapy to each patient (precision therapy) (91). To these ends, we envision routine dosimetry based on individualized internal organ geometries and rates of drug uptake and clearance. Such effort may include, but is not limited to, developing streamlined dosimetry methods based on patient-specific, image-based radiopharmaceutical distribution rather than previous-generation geometric models; optimizing the frequency and timing of SPECT/CT or planar imaging studies, as needed, to enable accurate personalized dosimetric calculations; and advancing microdosimetry methods for cellular or DNA-based dosimetry models to support an understanding of radiobiologic effectiveness at the cellular and subcellular levels. Such efforts will be critical in optimized development of $\alpha$-particle-based therapies for which current dosimetry methods are inappropriate or limited.

\section{Combination of Radionuclide Therapy with Other Forms of Cancer Therapy}

MTRT will become a standard therapeutic approach used in combination with more standard cancer treatments at earlier tumor stages. So far, MTRT has been almost exclusively used as monotherapy in patients with very advanced disease and few or no other treatment options (92). Since the efficacy and safety of targeted radionuclide therapy has now been demonstrated unequivocally in this setting $(93,94)$, there is enormous potential in using targeted radionuclide therapy at early tumor stages and in combining targeted radionuclide therapy with other forms of cancer therapy in order to increase the frequency and duration of tumor responses. In addition, efficacy and safety may be increased by combining 2 radiopharmaceuticals with different biodistributions or intratumoral distributions. Substantial and clinically impactful research opportunities exist in this space.

\section{Mechanisms for Radiation-Induced Cytotoxicity}

Improvements in understanding the radiobiology of MTRT will inform optimized dosing strategies to better predict response and enhance treatment effects. Although patient-specific dosimetry is likely to explain some of the variability of clinical responses to targeted radionuclide therapy, there are many clinical cases of exceptional responses without a particularly high tumor uptake of the radiopharmaceutical. Conversely, patients may not respond despite high tumor uptake. Possible explanations include heterogeneous 
expression of the target in different lesions (intermetastatic and intrametastatic heterogeneity) (95), inhomogeneous distribution of the radiopharmaceutical within a lesion because of differences in regional perfusion (96), presence or absence of DNA repair defects, and immunologic effects caused by radionuclide therapy. Systematic studies of these factors are scientifically highly interesting and will likely result in the identification of new rational combinations of radionuclide therapy with other targeted drugs. A much greater understanding of the radiobiology of MTRT will be essential, informed by the most modern scientific methods.

\section{High-Quality Clinical Trials of MTRT}

Carefully designed prospective trials comparing the efficacy of MTRT with that of conventional therapies will demonstrate clinical benefits, quality-of-life improvements, and medicoeconomic efficiencies. One of the limitations to the adoption of some NM methods has been a lack of high-quality prospective clinical data. For MTRT to be fully accepted, well-designed prospective clinical trials must be conducted in which the MTRT agents are directly compared with more standard therapies in a randomized fashion. This was done in the development of ${ }^{223} \mathrm{RaCl}_{2}$ (radium) and in the development of ${ }^{177} \mathrm{Lu}$-DOTATATE. Such definitive trials are essential to moving the field ahead, to securing suitable reimbursement, and to achieving dissemination of the methods. Careful trials that show the value of dosimetry as compared with more empiric approaches will be essential. Tracking patient-reported quality of life outcomes and actual costs in delivering patient care will be important as well, to lift nuclear oncology practice to the evidence level of medical, radiation, or surgical oncology.

\section{CONCLUSION}

There are many exceptional opportunities for research and discovery in NM. In instrumentation, new instruments and data analysis approaches are emerging with the potential to drive new applications and improve old ones. For new agents in cardiac, neurologic, and oncology areas, the fundamental challenge for MI is appropriate and thorough characterization of existing and novel compounds, with a focus on informing therapeutic strategies and documenting associated clinical outcomes. It is essential to identify high-reward candidate tracers early in the pipeline and to demonstrate sufficient versatility of applications to facilitate clinical translation. Reliable and reproducible quantification for wider application is requisite to demonstrate independent prognostic value and detection of abnormal expression; quantification is an MI strength, and this must remain a priority. Finally, optimal tracers should distinguish not only the presence and severity of disease but also a reduction of signal in response to effective treatment, which necessitates high signal strength and reproducibility of measurement. For MTRT, better dosimetry, radiobiology, and high-quality clinical trials represent high-impact opportunities. These could be accelerated by major investments in the field.

Finally, the role of the NM physician in the treatment of a patient must evolve. To realize the potential of precision medicine, $\mathrm{NM}$ and MI data must be integrated with other patient data and with the NM physician as key player on the patient management team. This is especially true as the role of MTRT is expanded into new cancer areas and earlier disease states. Training for physicians and the entire NM and MI team will need to evolve to the meet the needs of the NM and MI department of the near future.

\section{DISCLOSURE}

Richard Wahl is a consultant and on the scientific advisory board for Clarity Pharmaceuticals. Panithaya Chareonthaitawee is a consultant for Bioclinica, GE Healthcare, and MedTrace and receives royalties from UpToDate. Alexander Drzezga receives research support from Siemens Healthineers, Life Molecular Imaging, GE Healthcare, and AVID Radiopharmaceuticals; is on the speaker honorary/advisory boards for Siemens Healthineers, Sanofi, and GE Healthcare; holds stock in Siemens Healthineers; and has a patent pending for ${ }^{18}$ F-PSMA7 (PSMA PET imaging tracer). Gary Ulaner is a consultant for Sanofi and receives grant support from Sanofi, Novartis, Genentech, and Puma Biotechnology. Wolfgang Weber is on the advisory boards of, and receives compensation from, Bayer, Blue Earth Diagnostics, Endocyte, ITM, and Pentixapharm and receives research support from BMS, ImaginAb, Ipsen, and Piramal. No other potential conflict of interest relevant to this article was reported.

\section{ACKNOWLEDGMENTS}

We acknowledge the Society of Nuclear Medicine and Molecular Imaging council and center members who contributed with their ideas to this article.

\section{REFERENCES}

1. Chen JH, Asch SM. Machine learning and prediction in medicine: beyond the peak of inflated expectations. N Engl J Med. 2017;376:2507-2509.

2. Hatt M, Tixier F, Pierce L, Kinahan PE, Le Rest CC, Visvikis D. Characterization of PET/CT images using texture analysis: the past, the present... any future? Eur J Nucl Med Mol Imaging. 2017;44:151-165.

3. Minoshima S, Frey KA, Koeppe RA, Foster NL, Kuhl DE. A diagnostic approach in Alzheimer's disease using three-dimensional stereotactic surface projections of fluorine-18-FDG PET. J Nucl Med. 1995;36:1238-1248.

4. Ohliger MA, Hope TA, Chapman JS, Chen LM, Behr SC, Poder L. PET/MR imaging in gynecologic oncology. Magn Reson Imaging Clin N Am. 2017;25: $667-684$.

5. Oldan JD, Shah SN, Rose TL. Applications of PET/MR imaging in urogynecologic and genitourinary cancers. Magn Reson Imaging Clin N Am. 2017;25: $335-350$.

6. Cherry SR, Jones T, Karp JS, Qi JY, Moses WW, Badawi RD. Total-body PET: maximizing sensitivity to create new opportunities for clinical research and patient care. J Nucl Med. 2018;59:3-12.

7. Saboury B, Morris MA, Farhadi F, et al. Reinventing molecular imaging with total-body PET, part I: technical revolution in evolution. PET Clin. 2020;15: 427-438.

8. Borson S, Frank L, Bayley PJ, et al. Improving dementia care: the role of screening and detection of cognitive impairment. Alzheimers Dement. 2013;9: 151-159.

9. Dimitrakopoulou-Strauss A, Pan L, Strauss LG. Parametric imaging: a promising approach for the evaluation of dynamic PET- ${ }^{18}$ F-FDG studies- the DKFZ experience. Hell J Nucl Med. 2010;13:18-22.

10. Rahmim A, Lodge MA, Karakatsanis NA, et al. Dynamic whole-body PET imaging: principles, potentials and applications. Eur J Nucl Med Mol Imaging. 2019;46:501-518.

11. Herholz K. FDG PET and differential diagnosis of dementia. Alzheimer Dis Assoc Disord. 1995;9:6-16.

12. Minoshima S, Foster NL, Sima AA, Frey KA, Albin RL, Kuhl DE. Alzheimer's disease versus dementia with Lewy bodies: cerebral metabolic distinction with autopsy confirmation. Ann Neurol. 2001;50:358-365.

13. Rathore C, Dickson JC, Teotonio R, Ell P, Duncan JS. The utility of ${ }^{18}$ F-fluorodeoxyglucose PET (FDG PET) in epilepsy surgery. Epilepsy Res. 2014;108: 1306-1314.

14. Sha SJ, Ghosh PM, Lee SE, et al. Predicting amyloid status in corticobasal syndrome using modified clinical criteria, magnetic resonance imaging and fluorodeoxyglucose positron emission tomography. Alzheimers Res Ther. 2015;7:8.

15. Tahmasian M, Pasquini L, Scherr M, et al. The lower hippocampus global connectivity, the higher its local metabolism in Alzheimer disease. Neurology. 2015;84:1956-1963. 
16. Perneczky R, Diehl-Schmid J, Drzezga A, Kurz A. Brain reserve capacity in frontotemporal dementia: a voxel-based ${ }^{18}$ F-FDG PET study. Eur J Nucl Med Mol Imaging. 2007;34:1082-1087.

17. Cai Z, Li S, Matuskey D, Nabulsi N, Huang Y. PET imaging of synaptic density: a new tool for investigation of neuropsychiatric diseases. Neurosci Lett. 2019;691: 44-50.

18. Chen MK, Mecca AP, Naganawa M, et al. Assessing synaptic density in Alzheimer disease with synaptic vesicle glycoprotein $2 \mathrm{~A}$ positron emission tomographic imaging. JAMA Neurol. 2018;75:1215-1224.

19. Constantinescu CC, Tresse C, Zheng M, et al. Development and in vivo preclinical imaging of fluorine-18-labeled synaptic vesicle protein 2A (SV2A) PET tracers. Mol Imaging Biol. 2019;21:509-518.

20. Drzezga A. Amyloid-plaque imaging in early and differential diagnosis of dementia. Ann Nucl Med. 2010;24:55-66.

21. Villemagne VL, Dore V, Burnham SC, Masters CL, Rowe CC. Imaging tau and amyloid-beta proteinopathies in Alzheimer disease and other conditions. Nat Rev Neurol. 2018;14:225-236.

22. Bischof GN, Endepols H, van Eimeren T, Drzezga A. Tau-imaging in neurodegeneration. Methods. 2017;130:114-123.

23. Fleisher AS, Pontecorvo MJ, Devous MD Sr, et al. Positron emission tomography imaging with $\left[{ }^{18} \mathrm{~F}\right]$ flortaucipir and postmortem assessment of Alzheimer disease neuropathologic changes. JAMA Neurol. 2020;77:829-839.

24. Alam MM, Lee J, Lee SY. Recent progress in the development of TSPO PET ligands for neuroinflammation imaging in neurological diseases. Nucl Med $\mathrm{Mol}$ Imaging. 2017;51:283-296.

25. Janssen B, Vugts DJ, Windhorst AD, Mach RH. PET imaging of microglial activation-beyond targeting TSPO. Molecules. 2018;23:607.

26. Kim MJ, Shrestha SS, Cortes M, et al. Evaluation of two potent and selective PET radioligands to image COX-1 and COX-2 in rhesus monkeys. J Nucl Med. 2018;59:1907-1912.

27. Cummings JL, Henchcliffe C, Schaier S, Simuni T, Waxman A, Kemp P. The role of dopaminergic imaging in patients with symptoms of dopaminergic system neurodegeneration. Brain. 2011;134:3146-3166.

28. Suwijn SR, van Boheemen CJ, de Haan RJ, Tissingh G, Booij J, de Bie RM. The diagnostic accuracy of dopamine transporter SPECT imaging to detect nigrostriatal cell loss in patients with Parkinson's disease or clinically uncertain parkinsonism: a systematic review. EJNMMI Res. 2015;5:12.

29. Majo VJ, Prabhakaran J, Mann JJ, Kumar JS. PET and SPECT tracers for glutamate receptors. Drug Discov Today. 2013;18:173-184.

30. Rischpler C, Dirschinger RJ, Nekolla SG, et al. Prospective evaluation of ${ }^{18} \mathrm{~F}-$ fluorodeoxyglucose uptake in postischemic myocardium by simultaneous positron emission tomography/magnetic resonance imaging as a prognostic marker of functional outcome. Circ Cardiovasc Imaging. 2016;9:e004316.

31. Thackeray JT, Bengel FM. Molecular imaging of myocardial inflammation with positron emission tomography post-ischemia: a determinant of subsequent remodeling or recovery. JACC Cardiovasc Imaging. 2018;11:1340-1355.

32. Hess A, Thackeray JT, Wollert KC, Bengel FM. Radionuclide image-guided repair of the heart. JACC Cardiovasc Imaging. December 18, 2019 [Epub ahead of print].

33. Varasteh Z, Mohanta S, Robu S, et al. Molecular imaging of fibroblast activity after myocardial infarction using a ${ }^{68} \mathrm{Ga}$-labeled fibroblast activation protein inhibitor, FAPI-04. J Nucl Med. 2019;60:1743-1749.

34. Nahrendorf M, Frantz S, Swirski FK, et al. Imaging systemic inflammatory networks in ischemic heart disease. J Am Coll Cardiol. 2015;65:1583-1591.

35. Slomka PJ, Betancur J, Liang JX, et al. Rationale and design of the REgistry of Fast Myocardial Perfusion Imaging with NExt generation SPECT (REFINE SPECT). J Nucl Cardiol. 2020;27:1010-1021.

36. Wells RG, Marvin B, Poirier M, Renaud J, deKemp RA, Ruddy TD. Optimization of SPECT measurement of myocardial blood flow with corrections for attenuation, motion, and blood binding compared with PET. J Nucl Med. 2017;58: 2013-2019.

37. Dilsizian V, Taillefer R. Journey in evolution of nuclear cardiology: will there be another quantum leap with the F-18-labeled myocardial perfusion tracers? JACC Cardiovasc Imaging. 2012;5:1269-1284.

38. Bravo PE, Dorbala S. Targeted nuclear imaging probes for cardiac amyloidosis. Curr Cardiol Rep. 2017;19:59.

39. Gillmore JD, Maurer MS, Falk RH, et al. Nonbiopsy diagnosis of cardiac transthyretin amyloidosis. Circulation. 2016;133:2404-2412.

40. Gowrishankar G, Hardy J, Wardak M, et al. Specific imaging of bacterial infection using $6{ }^{\prime \prime}-{ }^{18} \mathrm{~F}$-fluoromaltotriose: a second-generation PET tracer targeting the maltodextrin transporter in bacteria. J Nucl Med. 2017;58:1679-1684.

41. Jadvar H, Colletti PM, Delgado-Bolton R, et al. Appropriate use criteria for ${ }^{18} \mathrm{~F}-$ FDG PET/CT in restaging and treatment response assessment of malignant disease. J Nucl Med. 2017;58:2026-2037.
42. FDA approves ${ }^{11} \mathrm{C}$-choline for PET in prostate cancer. $J$ Nucl Med. 2012;53(12): $11 \mathrm{~N}$.

43. FDA approves ${ }^{18} \mathrm{~F}$-fluciclovine and ${ }^{68} \mathrm{Ga}$-DOTATATE products. $\mathrm{J}$ Nucl Med. 2016;57(8):9N.

44. Gaur S, Mena E, Harmon SA, et al. Prospective evaluation of ${ }^{18}$ F-DCFPyL PET/ $\mathrm{CT}$ in detection of high-risk localized prostate cancer: comparison with mpMRI. AJR. 2020;215:1-8.

45. Lindenberg L, Mena E, Turkbey B, et al. Evaluating biochemically recurrent prostate cancer: histologic validation of ${ }^{18} \mathrm{~F}-\mathrm{DCFPyL}$ PET/CT with comparison to multiparametric MRI. Radiology. 2020;296:564-572.

46. Tan N, Oyoyo U, Bavadian N, et al. PSMA-targeted radiotracers versus ${ }^{18} \mathrm{~F}$ fluciclovine for the detection of prostate cancer biochemical recurrence after definitive therapy: a systematic review and meta-analysis. Radiology. 2020;296: 44-55.

47. Calais J, Ceci F, Eiber M, et al. ${ }^{18}$ F-fluciclovine PET-CT and ${ }^{68}$ Ga-PSMA-11 PET-CT in patients with early biochemical recurrence after prostatectomy: a prospective, single-centre, single-arm, comparative imaging trial. Lancet Oncol. 2019;20:1286-1294.

48. Sonni I, Eiber M, Fendler WP, et al. Impact of ${ }^{68}$ Ga-PSMA-11 PET/CT on staging and management of prostate cancer patients in various clinical settings: a prospective single-center study. J Nucl Med. 2020;61:1153-1160.

49. Calais J, Kishan AU, Cao M, et al. Potential impact of ${ }^{68} \mathrm{Ga}$-PSMA-11 PET/CT on the planning of definitive radiation therapy for prostate cancer. $J$ Nucl Med. 2018;59:1714-1721.

50. Katzenellenbogen JA. The quest for improving the management of breast cancer by functional imaging: the discovery and development of $16 \alpha-\left[{ }^{18} \mathrm{~F}\right]$ fluoroestradiol (FES), a PET radiotracer for the estrogen receptor, a historical review. Nucl Med Biol. February 22, 2020 [Epub ahead of print].

51. Dehdashti F, Mortimer JE, Trinkaus K, et al. PET-based estradiol challenge as a predictive biomarker of response to endocrine therapy in women with estrogenreceptor-positive breast cancer. Breast Cancer Res Treat. 2009;113:509-517.

52. Mortimer JE, Dehdashti F, Siegel BA, Trinkaus K, Katzenellenbogen JA, Welch MJ. Metabolic flare: indicator of hormone responsiveness in advanced breast cancer. J Clin Oncol. 2001;19:2797-2803.

53. Ulaner GA, Jhaveri K, Chardarlapaty S, et al. Head-to-head evaluation of ${ }^{18} \mathrm{~F}$ FES and ${ }^{18} \mathrm{~F}-\mathrm{FDG}$ PET/CT in metastatic invasive lobular breast cancer. $\mathrm{J} \mathrm{Nucl}$ Med. July 17, 2020 [Epub ahead of print].

54. Hogan MP, Goldman DA, Dashevsky B, et al. Comparison of ${ }^{18}$ F-FDG PET/CT for systemic staging of newly diagnosed invasive lobular carcinoma versus invasive ductal carcinoma. J Nucl Med. 2015;56:1674-1680.

55. Kurland BF, Wiggins JR, Coche A, et al. Whole-body characterization of estrogen receptor status in metastatic breast cancer with $16 \alpha-{ }^{18} \mathrm{~F}$-fluoro- $17 \beta$-estradiol positron emission tomography: meta-analysis and recommendations for integration into clinical applications. Oncologist. May 6, 2020 [Epub ahead of print].

56. Sun Y, Yang Z, Zhang Y, et al. The preliminary study of $16 \alpha-\left[{ }^{18} \mathrm{~F}\right]$ fluoroestradiol $\mathrm{PET} / \mathrm{CT}$ in assisting the individualized treatment decisions of breast cancer patients. PLoS One. 2015;10:e0116341.

57. Chan SR, Fowler AM, Allen JA, et al. Longitudinal noninvasive imaging of progesterone receptor as a predictive biomarker of tumor responsiveness to estrogen deprivation therapy. Clin Cancer Res. 2015;21:1063-1070.

58. Fowler AM, Clark AS, Katzenellenbogen JA, Linden HM, Dehdashti F. Imaging diagnostic and therapeutic targets: steroid receptors in breast cancer. J Nucl Med. 2016;57(suppl 1):75S-80S.

59. Hoefnagel LD, van de Vijver MJ, van Slooten HJ, et al. Receptor conversion in distant breast cancer metastases. Breast Cancer Res. 2010;12:R75.

60. Niikura N, Liu J, Hayashi N, et al. Loss of human epidermal growth factor receptor 2 (HER2) expression in metastatic sites of HER2-overexpressing primary breast tumors. J Clin Oncol. 2012;30:593-599.

61. Priedigkeit N, Hartmaier RJ, Chen $\mathrm{Y}$, et al. Intrinsic subtype switching and acquired ERBB2/HER2 amplifications and mutations in breast cancer brain metastases. JAMA Oncol. 2017;3:666-671.

62. Henry KE, Ulaner GA, Lewis JS. Human epidermal growth factor receptor 2targeted PET/single-photon emission computed tomography imaging of breast cancer: noninvasive measurement of a biomarker integral to tumor treatment and prognosis. PET Clin. 2017;12:269-288.

63. Ulaner GA, Hyman DM, Ross DS, et al. Detection of HER2-positive metastases in patients with HER2-negative primary breast cancer using ${ }^{89} \mathrm{Zr}$-trastuzumab PET/CT. J Nucl Med. 2016;57:1523-1528.

64. Ulaner GA, Lyashchenko SK, Riedl C, et al. First-in-human human epidermal growth factor receptor 2-targeted imaging using ${ }^{89} \mathrm{Zr}$-pertuzumab PET/CT: dosimetry and clinical application in patients with breast cancer. J Nucl Med. 2018;59: 900-906.

65. Ulaner GA, Hyman DM, Lyashchenko SK, Lewis JS, Carrasquillo JA. ${ }^{89} \mathrm{Zr}$ trastuzumab PET/CT for detection of human epidermal growth factor receptor 
2-positive metastases in patients with human epidermal growth factor receptor 2negative primary breast cancer. Clin Nucl Med. 2017;42:912-917.

66. Ulaner GA, Carrasquillo JA, Riedl CC, et al. Identification of HER2-positive metastases in patients with HER2-negative primary breast cancer by using HER2-targeted ${ }^{89}$ Zr-pertuzumab PET/CT. Radiology. 2020;296:370-378.

67. Shi X, Xing H, Yang X, et al. Fibroblast imaging of hepatic carcinoma with ${ }^{68} \mathrm{Ga}$-FAPI-04 PET/CT: a pilot study in patients with suspected hepatic nodules. Eur J Nucl Med Mol Imaging. May 29, 2020 [Epub ahead of print].

68. Syed M, Flechsig P, Liermann J, et al. Fibroblast activation protein inhibitor (FAPI) PET for diagnostics and advanced targeted radiotherapy in head and neck cancers. Eur J Nucl Med Mol Imaging. May 23, 2020 [Epub ahead of print].

69. Toms J, Kogler J, Maschauer S, et al. Targeting fibroblast activation protein: radiosynthesis and preclinical evaluation of an ${ }^{18} \mathrm{~F}$-labeled FAP inhibitor. $\mathrm{J} \mathrm{Nucl}$ Med. April 24, 2020 [Epub ahead of print].

70. Kratochwil C, Flechsig P, Lindner T, et al. ${ }^{68}$ Ga-FAPI PET/CT: tracer uptake in 28 different kinds of cancer. J Nucl Med. 2019;60:801-805.

71. Loktev A, Lindner T, Burger EM, et al. Development of fibroblast activation protein-targeted radiotracers with improved tumor retention. $J$ Nucl Med. 2019;60: 1421-1429.

72. Giesel FL, Kratochwil C, Lindner T, et al. ${ }^{68}$ Ga-FAPI PET/CT: biodistribution and preliminary dosimetry estimate of 2 DOTA-containing FAP-targeting agents in patients with various cancers. J Nucl Med. 2019;60:386-392.

73. Caserta E, Chea J, Minnix M, et al. Copper 64-labeled daratumumab as a PET/ CT imaging tracer for multiple myeloma. Blood. 2018;131:741-745.

74. Ghai A, Maji D, Cho N, et al. Preclinical development of CD38-targeted [ $\left.{ }^{89} \mathrm{Zr}\right] \mathrm{Zr}-$ DFO-daratumumab for imaging multiple myeloma. J Nucl Med. 2018;59:216-222.

75. Kang L, Jiang D, England CG, et al. ImmunoPET imaging of CD38 in murine lymphoma models using ${ }^{89} \mathrm{Zr}$-labeled daratumumab. Eur J Nucl Med Mol Imaging. 2018;45:1372-1381.

76. Ulaner GA, Sobol NB, O'Donoghue JA, et al. CD38-targeted immuno-PET of multiple myeloma: from xenograft models to first-in-human imaging. Radiology. 2020;295:606-615.

77. Pandit-Taskar N, Zanzonico P, Staton KD, et al. Biodistribution and dosimetry of ${ }^{18}$ F-meta-fluorobenzylguanidine: a first-in-human PET/CT imaging study of patients with neuroendocrine malignancies. J Nucl Med. 2018;59:147-153.

78. Cho SY, Lipson EJ, Im HJ, et al. Prediction of response to immune checkpoint inhibitor therapy using early-time-point ${ }^{18} \mathrm{~F}-\mathrm{FDG}$ PET/CT imaging in patients with advanced melanoma. $J$ Nucl Med. 2017;58:1421-1428.

79. Bensch F, van der Veen EL, Lub-de Hooge MN, et al. ${ }^{89} \mathrm{Zr}$-atezolizumab imaging as a non-invasive approach to assess clinical response to PD-L1 blockade in cancer. Nat Med. 2018;24:1852-1858.

80. Colevas D, Bedi N, Chang S, et al. A study to evaluate immunological response to PD-1 inhibition in squamous cell carcinoma of the head and neck (SCCHN) using novel PET imaging with $\left[{ }^{18} \mathrm{~F}\right] \mathrm{F}-\mathrm{AraG}$ [abstract $]$. J Clin Oncol. 2018;36:6050.
81. Tavaré R, Escuin-Ordinas H, Mok S, et al. An effective immuno-PET imaging method to monitor CD8-dependent responses to immunotherapy. Cancer Res. 2016;76:73-82.

82. Pandit-Taskar N, Postow MA, Hellmann MD, et al. First-in-humans imaging with ${ }^{89} \mathrm{Zr}$-Df-IAB22M2C anti-CD8 minibody in patients with solid malignancies: preliminary pharmacokinetics, biodistribution, and lesion targeting. J Nucl Med. 2020;61:512-519.

83. Barrio M, Czernin J, Fanti S, et al. The impact of somatostatin receptor-directed PET/CT on the management of patients with neuroendocrine tumor: a systematic review and meta-analysis. J Nucl Med. 2017;58:756-761.

84. Strosberg J, Krenning E. ${ }^{177}$ Lu-dotatate for midgut neuroendocrine tumors. $N$ Engl J Med. 2017;376:1391-1392.

85. Eiber M, Fendler WP, Rowe SP, et al. Prostate-specific membrane antigen ligands for imaging and therapy. J Nucl Med. 2017;58(suppl):67S-76S.

86. Han S, Woo S, Kim YJ, Suh CH. Impact of ${ }^{68}$ Ga-PSMA PET on the management of patients with prostate cancer: a systematic review and meta-analysis. Eur Urol. 2018;74:179-190.

87. Virgolini I, Decristoforo C, Haug A, Fanti S, Uprimny C. Current status of theranostics in prostate cancer. Eur J Nucl Med Mol Imaging. 2018;45:471-495.

88. Zschaeck S, Lohaus F, Beck M, et al. PSMA-PET based radiotherapy: a review of initial experiences, survey on current practice and future perspectives. Radiat Oncol. 2018;13:90.

89. Langbein T, Weber WA, Eiber M. Future of theranostics: an outlook on precision oncology in nuclear medicine. J Nucl Med. 2019;60(suppl):13S-19S.

90. Dewaraja YK, Frey EC, Sgouros G, et al. MIRD pamphlet no. 23: quantitative SPECT for patient-specific 3-dimensional dosimetry in internal radionuclide therapy. J Nucl Med. 2012;53:1310-1325.

91. Del Prete M, Buteau FA, Beauregard JM. Personalized ${ }^{177}$ Lu-octreotate peptide receptor radionuclide therapy of neuroendocrine tumours: a simulation study. Eur J Nucl Med Mol Imaging. 2017;44:1490-1500.

92. Claringbold PG, Brayshaw PA, Price RA, Turner JH. Phase II study of radiopeptide ${ }^{177} \mathrm{Lu}$-octreotate and capecitabine therapy of progressive disseminated neuroendocrine tumours. Eur J Nucl Med Mol Imaging. 2011;38:302-311.

93. Parker C, Nilsson S, Heinrich D, et al. Alpha emitter radium-223 and survival in metastatic prostate cancer. N Engl J Med. 2013;369:213-223.

94. Strosberg J, El-Haddad G, Wolin E, et al. Phase 3 trial of ${ }^{177} \mathrm{Lu}$-dotatate for midgut neuroendocrine tumors. N Engl J Med. 2017;376:125-135.

95. Hofman MS, Violet J, Hicks RJ, et al. $\left[{ }^{177} \mathrm{Lu}\right]-$ PSMA-617 radionuclide treatment in patients with metastatic castration-resistant prostate cancer (LuPSMA trial): a single-centre, single-arm, phase 2 study. Lancet Oncol. 2018;19:825-833.

96. Falzone N, Lee BQ, Able S, et al. Targeting micrometastases: the effect of heterogeneous radionuclide distribution on tumor control probability. $\mathrm{J} \mathrm{Nucl}$ Med. 2018;60:250-258. 